\title{
DUAL GINZBURG-LANDAU THEORY AND CHIRAL SYMMETRY BREAKING
}

\author{
Hiroshi TOKI*, Shoichi SASAKI and Hideo SUGANUMA \\ RCNP, Osaka University,Ibaraki,Osaka 567,Japan
}

\begin{abstract}
We study the properties of quarks, being confined in hadrons, with the Schwinger-Dyson equation in the dual Ginzburg-Landau Theory. Magnetic monopole condensation, which provides quark confinement, is demonstrated responsible also for dynamical chiral-symmetry breaking. We discuss then the recovery of the chiral symmetry at finite temperature.
\end{abstract}

\section{Introduction}

Quarks are not found in the free space, while they are seen by deep inelastic scattering in hadrons. For the hadron physics, it is the essential problem to understand why and how quarks are confined in hadrons. Since quark confinement is not understood, there are many popular and sometimes conflicting models for nucleon suggested in the literature. In the bag picture, massless quarks are confined within the bag in the Wigner mode, while quarks are repelled and mesons are present outside the bag in the Nambu-Goldstone mode. Depending on the strength of confinement, from weak to strong, we have the MIT bag model, the chiral bag model and the Skyrmion model, respectively. In the constituent quark picture, quarks are massive and considered quasi-particle and they are confined by the long range confining potential. In this picture, confinement is not sharp but is gradually increasing with distance. We should understand the mechanism of quark confinement to settle our picture on nucleons and eventually on entire hadrons.

The chiral symmetry and its spontaneous breaking is also the essential feature of the hadron physics. In the light quark system, the QCD lagrangian possesses the chiral symmetry, since we can neglect safely the small current quark mass as compared to the nucleon mass scale. If this symmetry is kept, we expect parity doublets in hadron spectra. This is not the case by observing the meson spectrum as the pion $\left(0^{-}\right)$has an extremely small mass and the parity partner, the sigma $\left(0^{+}\right)$meson, is not found, and the rho $\left(1^{-}\right)$and the $A_{1}\left(1^{+}\right)$mesons do have completely different masses by about $450 \mathrm{MeV}$. Confinement of quarks and chiral symmetry breaking are most important phenomena in the hadron physics.

The dynamics of the hadron physics are believed to be described by QCD. In the perturbative QCD, the renormalized gauge coupling constant shows the asymptotic free property at large momentum, $Q$, while it becomes progressively large at small

*e-mail toki@miho.rcnp.osaka-u.ac.jp 
$Q$, where confinement and chiral symmetry breaking are expected. What is essential here at small $Q$ ?

Here, the lattice QCD theory demonstrates quark confinement, chiral symmetry breaking, hadron masses and many other nonperturbative properties. The recent simulation studies have shown that the essential degree of freedom for these phenomena is QCD-monopole and its condensation. There are many talks on the importance of the QCD monopole field in Confinement'95.

\section{Dual Ginzburg-Landau Theory}

What is then the QCD-monopoles? 't Hooft has demonstrated that the nonabelian gauge theory as QCD is reduced to an abelian gauge theory as QED with magnetic monopoles by abelian gauge fixing, which is a choice of a particular gaugel. Suzuki and his collaborators in the Kanazawa group, have then discussed extensively the dual Ginzburg-Landau (DGL) theory $\mathrm{A}$. In the DGL Theory, the non-abelian part is considered negligible and the QCD-monopole field couples with the dual gauge field. The biggest assumption is then the introduction of the self interaction term of QCD-monopoles, which is introduced by hand, to cause QCD-monopole condensation and then the dual Higgs mechanism, providing a finite mass, $m_{B}$, to the dual gauge field. The details of the DGL theory are provided in the papers of Matsubara and Suganuma et.al. in Confinement'95 5 国.

We can then imitate the dual Meissner effect for quark confinement as Nambul has demonstrated it just after Nielsen and Olesen 1 have formulated the relativistic version of the Ginzburg-Landau theory on the vortex solution in superconductors. We only reverse the roles of the electric and the magnetic fields, which is called dual transformation, hence the word dual Ginzburg-Landau theory. When the dual gauge field gets a mass, the color electric flux cannot spread out freely from one source to the other and is confined in a vortex-like configuration, which resembles the confinement of the magnetic field in the Abrikosov vortex in the superconductor.

\section{Static Confining Potential}

It is straightforward to calculate the static confining potential using the DGL lagrangian by putting a quark and an anti-quark separated by a distance $r$.

$$
\vec{j}_{\mu}(x)=\vec{Q} g_{\mu 0}\left\{\delta^{3}(\mathbf{x}-\mathbf{b})-\delta^{3}(\mathbf{x}-\mathbf{a})\right\} .
$$

where $\mathbf{r}=\mathbf{b}-\mathbf{a}$. This leads to the static potential as

$$
\begin{aligned}
V(r) & =\vec{Q}^{2} \int \frac{d^{3} k}{(2 \pi)^{3}} \frac{1}{2}\left(1-e^{i \mathbf{k} \cdot \mathbf{r}}\right)\left(1-e^{-i \mathbf{k} \cdot \mathbf{r}}\right)\left[\frac{1}{\mathbf{k}^{2}+m_{B}^{2}}+\frac{m_{B}^{2}}{\mathbf{k}^{2}+m_{B}^{2}} \frac{n^{2}}{(\mathbf{n} \cdot \mathbf{k})}\right] \\
& =-\frac{\vec{Q}^{2}}{4 \pi} \cdot \frac{e^{-m_{B} r}}{r}+\frac{\vec{Q}^{2} m_{B}^{2}}{8 \pi} \ln \left(\frac{m_{B}^{2}+m_{\chi}^{2}}{m_{B}^{2}}\right) r,
\end{aligned}
$$


besides the $r$-independent term for the case of type-II dual superconductor $\left(m_{B}<\right.$ $\left.m_{\chi}\right)$. We get the finite expression because of the following the points, ; i.e. the direction of $\mathbf{n}$ to be parallel to $\mathbf{r}(\mathbf{n} / / \mathbf{r})$ due to the energy minimum condition and the axial symmetry of the system, and the ultraviolet cutoff appears in the perpendicular direction in $k_{T}$ integration $\left(k_{T}<m_{\chi}\right)$ according to the vanishing of the QCD-monopole field at the center of the vortex-like configuration outward. The Yukawa term provides a short range attraction and the linear term a long range confining potentialt.

The potential may be adjusted to the phenomenological potential of the Cornell group and the parameters are extracted by the fitting procedure $\mathrm{B}$. This procedure provides the masses of the glueballs, which are associated with the properties of the QCD vacuum and quark confinement. It is very important to find these glueballs with masses around $1 \mathrm{GeV}$ by experiment.

\section{Dynamical Chiral-Symmetry Breaking}

We would like now to discuss the behavior of quarks in the QCD-monopole condensed vacuum. This amounts to calculate the quark propagator using the Schwinger -Dyson equation. It seems, however, not straightforward, since quarks are confined. When we want to describe a quark, there are every time other quarks nearby and hence, in principle, we ought to treat the system of $q \bar{q}$ or $q q q$ or more complex ones with color singlet configurations totally.

This situation is similar to nuclear matter theory. In many-body nuclear system, each nucleon pair interacts strongly, in particular, the short range part of the interaction even diverges. Brueckner suggested first to solve a pair of nucleons, while the existence of all the other nucleons is considered to block the quantum states due to the Pauli effect. Hence, a nucleon pair interacts through the $T$-matrix in free space,

$$
T=V+V \frac{1}{e} T
$$

while a nucleon pair in the many body system interacts through the $G$-matrix with the Pauli exclusion operator $\hat{Q}$ as

$$
G=V+V \frac{\hat{Q}}{e} G
$$

This Pauli exclusion operator makes the effective interaction $G$ moderate in the medium. Then, we can make the Hatree-Fock procedure to calculate the total energy and the single particle properties of nucleon in the medium.

Coming back to the properties of quarks, which are confined, we ought to solve the Schwinger-Dyson equation,

$$
S^{-1}(p)=S_{0}^{-1}(p)+\int_{0}^{\infty} S(p-q) D(q) d q
$$


which are written schematically. $S$ and $S_{0}$ denote the full propagator and the simple propagator of a single quark, while $D$ the gluon propagator. This expression does not take care of quark confinement, however. Since quarks and gluons are confined inside of the hadrons, we have to introduce a low momentum cutoff $q_{c}$, which is associated with the size $R \sim q_{c}^{-1}$ of hadrons. Hence, the SD equation ought to be modified to

$$
S^{-1}(p)=S_{0}^{-1}(p)+\int_{q_{c}}^{\infty} S(p-q) D(q) d q
$$

Written rigorously, the Schwinger-Dyson equation in the DGL theory is

$$
S^{-1}(p)=i \not p+\int \frac{d^{4} q}{(2 \pi)^{4}} \vec{Q}^{2} \gamma^{\mu} S(p-q) \gamma^{\nu} D_{\mu \nu}(q)
$$

assuming the following simple form for the full quark propagator,

$$
S^{-1}(p)=i \not p-M\left(p^{2}\right)
$$

We find the integral equation for $M\left(p^{2}\right)$ as

$$
M\left(p^{2}\right)=\int \frac{d^{4} q}{(2 \pi)^{4}} \vec{Q}^{2} \frac{M\left((p-q)^{2}\right)}{(p-q)^{2}+M^{2}\left((p-q)^{2}\right)} D_{\mu}^{\mu}\left(q^{2}\right) .
$$

where the trace of the gluon propagator in the Landau gauge is

$$
D_{\mu}^{\mu}\left(q^{2}\right)=\frac{2}{q^{2}+m_{B}^{2}}+\frac{1}{q^{2}}+\frac{2}{(n \cdot q)^{2}+a^{2}} \cdot \frac{m_{B}^{2}}{q^{2}+m_{B}^{2}} .
$$

Here, we have introduced a infrared cutoff $a\left(\sim q_{c}\right)$, for the sake of numerical simplification, instead of the lower bound of the integration region.

We take further the angular average on the direction of the Dirac string because of light quarks moving in various directions inside of hadrons,

$$
\left\langle\frac{1}{(n \cdot q)^{2}+a^{2}}\right\rangle_{\text {average }} \equiv \frac{1}{2 \pi^{2}} \int d \Omega_{n} \frac{1}{(n \cdot q)^{2}+a^{2}}=\frac{1}{a} \cdot \frac{2}{a+\sqrt{a^{2}+q^{2}}} .
$$

We find the SD equation,

$$
\begin{aligned}
M\left(p^{2}\right)= & \int_{0}^{\infty} \frac{d k^{2}}{16 \pi^{2}} \frac{\vec{Q}^{2} M\left(k^{2}\right)}{k^{2}+M^{2}\left(k^{2}\right)}\left(\frac{4 k^{2}}{k^{2}+p^{2}+m_{B}^{2}+\sqrt{\left(k^{2}+p^{2}+m_{B}^{2}\right)^{2}-4 k^{2} p^{2}}}\right. \\
& +\frac{\left(1+\alpha_{e}\right) k^{2}}{\max \left(k^{2}, p^{2}\right)}+\frac{8 k^{2}}{\pi a} \int_{0}^{\pi} d \theta \frac{\sin ^{2} \theta}{a+\sqrt{k^{2}+p^{2}+a^{2}-2 k p \cos \theta}} \\
& \left.\times\left[\frac{m_{B}^{2}-a^{2}}{k^{2}+p^{2}+m_{B}^{2}-2 k p \cos \theta}+\frac{a^{2}}{k^{2}+p^{2}-2 k p \cos \theta}\right]\right) .
\end{aligned}
$$


where we have made the replacement, $k=p-q$.

We solve this $\mathrm{SD}$ equation numerically with the parameters ; $e=5.5, m_{B}=$ $0.5 \mathrm{GeV}$ and $a=85 \mathrm{MeV}$ with which the string tension is $k \simeq 1 \mathrm{GeV} / \mathrm{fm}$. We show

in Fig. 1 the quark mass $M\left(p^{2}\right)$ as a function of the Euclidean momentum in unit of $\Lambda_{\mathrm{QCD}}$. The glueball mass $m_{B}$ is proportional to the QCD-monopole condensate and $M\left(p^{2}\right)$ increases with $m_{B}$. This result indicates that the mechanism, which produces quark confinement, is able to cause chiral symmetry breaking as well. Concerning the condition for the parameters for the on-set of chiral symmetry breaking, we find with some approximation, the following simple relation,

$$
e^{2} m_{B}>24 \pi a
$$

This formula is very convenient to see that $m_{B}$ and hence QCD-monopole condensation plays the essential role for chiral symmetry breaking. Strictly speaking, the cutoff parameter $a$ depends on the glue ball mass, $m_{B}$, because $a$ is closely related with the confinement effect. With $m_{B}=0.5 \mathrm{GeV}$, we find the quark mass, the pion decay constant and also the quark condensate compare well with experimental values.

\section{Recovery of Chiral Symmetry at Finite temperature}

The chiral symmetry is broken at zero temperature, which is nicely described in the DGL theory. We then want to see if the theory provides the recovery of the chiral symmetry as the temperature increases. We take the imaginary-time formulation, and replace 


$$
\begin{aligned}
p_{4} & \rightarrow \omega_{n}=(2 n+1) \pi T \\
\int \frac{d^{4} k}{(2 \pi)^{4}} & \rightarrow T \sum_{m=-\infty}^{\infty} \int \frac{\mathrm{d} \mathbf{k}}{(2 \pi)^{3}} \\
M\left(p^{2}\right) & \rightarrow M_{T}\left(\omega_{n}, \mathbf{p}\right)
\end{aligned}
$$

Hence, the SD equation at finite temperature is formulated as

$$
\begin{aligned}
M_{T}\left(\omega_{n}, \mathbf{p}\right)= & T \sum_{m=-\infty}^{\infty} \int \frac{\mathrm{d} \mathbf{k}}{(2 \pi)^{3}} \vec{Q}^{2} \frac{M_{T}\left(\omega_{m}, \mathbf{k}\right)}{\mathbf{k}^{2}+\omega_{m}^{2}+M_{T}^{2}\left(\omega_{m}, \mathbf{k}\right)} \\
& {\left[\frac{2}{\tilde{k}_{n m}^{2}+m_{B}^{2}}+\frac{1}{\tilde{k}_{n m}^{2}}+\frac{4}{a} \frac{1}{a+\sqrt{\tilde{k}_{n m}^{2}+a^{2}}}\left(\frac{m_{B}^{2}-a^{2}}{\tilde{k}_{n m}^{2}+m_{B}^{2}}+\frac{a^{2}}{\tilde{k}_{n m}^{2}}\right)\right] }
\end{aligned}
$$

where $\tilde{k}_{n m}^{2}=\left(\omega_{n}-\omega_{m}\right)^{2}+(\mathbf{p}-\mathbf{k})^{2}$. This form is too difficult to solve exactly. Hence, we take the covariant-like ansatz for the self-energy at $T \neq 0$ as

$$
M_{T}\left(\omega_{n}, \mathbf{p}\right) \simeq M_{T}\left(\hat{p}^{2}\right) \text { with } \hat{p}^{2}=\mathbf{p}^{2}+\omega_{n}^{2}
$$

This assumption guarantees that the $\mathrm{SD}$ equation at $T \neq 0$ reduces to the one at $T=0$, when the angular average is taken in the three dimensional momentum space. We then find a manageable integral equation for $M_{T}\left(\hat{p}^{2}\right)$. We show in Fig. $2 M_{T}\left(\hat{p}^{2}\right)$ for various temperatures as a function of $\hat{p}^{2}$ in $\Lambda_{\mathrm{QCD}}$ unit. As expected, the mass function $M_{T}\left(\hat{p}^{2}\right)$ decreases rapidly with temperature. The quark condensate, $\langle\bar{q} q\rangle_{T}$, shown in Fig.3, decreases gradually with temperature in the low temperature region and vanishes suddenly near the critical temperature, in coincidence with recent lattice QCD simulations 10 . The critical temperature is found around $110 \mathrm{MeV}$. We should not take this value seriously though. All the parameters in the DGL lagrangian may be temperature dependent. In addition, the infrared cutoff parameter $a$ should be decreased with temperature, because the confinement effect gets weaker. We should also take into account the change of $m_{B}$ for further discussion of QCD phase transition at finite temperature日.

\section{Conclusion}

We have studied chiral symmetry breaking in terms of the dual Ginzburg-Landau (DGL) theory, which provides quark confinement. We solve the SD equation by introducing infrared cutoff $a$, which is due to the effect of the $q-\bar{q}$ pair creation and/or quark confinement. We then have found that the DGL theory can provide chiral symmetry breaking nicely with the parameters determined from the confining potential. We have demonstrated also that the DGL theory provides the recovery of the chiral symmetry at finite temperature. 
We stress again that QCD-monopole condensation, formulated in the DGL theory, causes both quark confinement and chiral symmetry breaking. We mention also that QCD-monopole condensation is signaled by the existence of glueballs with masses in the range of $1 \mathrm{GeV} ; m_{B}\left(1^{+}\right) \sim m_{\chi}\left(0^{+}\right) \sim 1 \mathrm{GeV}$, and hope that these glueballs are

found experimentally, since they are the key particles for the confinement mechanism due to QCD-monopole condensation 1 .

\section{Acknowledgment}

The authors are grateful to H. Ichie, H. Monden, T. Suzuki and O. Miyamura for fruitful discussions on the QCD-monopoles. 


\section{REFERENCES}

1. G. 't Hooft, Nucl. Phys. B190 (1981) 455.

2. T. Suzuki, Prog. Theor. Phys. 80 (1988) 929 ; 81 (1989) 752.

3. H. Suganuma, H. Ichie, S. Sasaki and H. Toki, this proceedings (1995).

4. Y. Matsubara, this proceedings (1995).

5. Y. Nambu, Phys. Rev. D10 (1974) 4262.

G. 't Hooft, in "High Energy Physics", ed. A. Zichichi (Editions Compositori, Bologna, 1975).

S. Mandelstam, Phys. Rep. D23 (1976) 245.

6. H. B. Nielsen and P. Olesen, Nucl. Phys. B61 (1973) 45.

7. H. Suganuma, S. Sasaki and H. Toki, Nucl. Phys. B435 (1995) 207.

8. S. Sasaki, H. Suganuma and H. Toki, Proceedings in "Quark Confinement and the Hadron Spectrum" (World Scientific, 1995) p.227.

9. H. Ichie, H. Suganuma and H. Toki, preprint RIKEN-AF-NP-176 (1994).

10. F. Karsch, this proceedings (1995). 